\title{
Antioxidant and Antiadipogenic Activities of Galkeun-Tang, a Traditional Korean Herbal Formula
}

\author{
Soo-Jin Jeong, Sae-Rom Yoo, Ohn-Soon Kim, Chang-Seob Seo, and Hyeun-Kyoo Shin \\ Herbal Medicine Formulation Research Group, Herbal Medicine Research Division, Korea Institute of Oriental Medicine, \\ Daejeon 305-811, Republic of Korea \\ Correspondence should be addressed to Hyeun-Kyoo Shin; hkshin@kiom.re.kr
}

Received 1 August 2014; Revised 18 November 2014; Accepted 23 November 2014; Published 10 December 2014

Academic Editor: Hyunsu Bae

Copyright (C) 2014 Soo-Jin Jeong et al. This is an open access article distributed under the Creative Commons Attribution License, which permits unrestricted use, distribution, and reproduction in any medium, provided the original work is properly cited.

\begin{abstract}
Galkeun-tang (GKT; Galgen-tang in Chinese and Kakkon-to in Japanese), a traditional herbal formula, has been used for treatment of the common cold. Here, we report in vitro antioxidant and antiadipogenic effects of GKT. GKT increased the activities of scavenging 2,2' -azinobis-(3-ethylbenzothiazoline-6-sulfonic acid) (ABTS) and 2,2-diphenyl-1-picrylhydrazyl (DPPH) radicals. GKT also significantly reduced the malondialdehyde (MDA) generation during low-density lipoprotein (LDL) oxidation and the electrophoretic mobility of oxidized LDL, indicating inhibitory effects of GKT on $\mathrm{Cu}^{2+}$-mediated oxidation of LDL. Regarding antiadipogenic activity, GKT treatment significantly suppressed lipid accumulation, triglyceride production, and glycerol-3phosphate dehydrogenase (GPDH) activity in differentiated 3T3-L1 adipocytes. Consistent with this, GKT significantly reduced the secretion of leptin, a major adipokine, in differentiated 3T3-L1 adipocytes. Overall, our findings suggest that GKT has the potential for antioxidative and antiadipogenic properties.
\end{abstract}

\section{Introduction}

Oxidative stress is an imbalance between the production of reactive oxygen species (ROS) and antioxidative defenses [1]. Disruption in normal redox signaling can mediate toxic effects and, thus, is closely associated with various human diseases [2]. Obesity is a metabolic disorder caused by excess fat accumulation in adipose tissue [3]. Recent papers have confirmed a critical role for oxidative stress in the pathogenesis of obesity or its associated diseases [4-6]. A change in protection against antioxidative mechanisms was observed in an obese rodent model and in obese human males [7, 8]. Thus, the development of antioxidants could be a valuable approach for treating and preventing obesity or its related diseases. Antioxidative activities of synthetic or natural products have been reported in obesity models. Natural products are considered particularly attractive antiobesity drug candidates because of their higher efficacies and fewer side effects.

Many traditional herbal medicines have attracted increasing attention for their complementary therapeutic effects with few or no side effects compared with Western medicines [9, 10]. Galkeun-tang (GKT; Galgen-tang in Chinese and
Kakkon-to in Japanese), a traditional herbal formula, has been used widely for treatment of the common cold, flu, and fever. In recent research papers, several pharmacological activities of GKT have been reported including antiviral, anti-inflammatory, and immune-regulating activities [11-15]. However, the effect of GKT on obesity or obesity-related diseases has not been reported. The pathogenesis of obesity and its associated diseases is closely related to an inflammatory reaction caused by nutrient excess and imbalance that has been termed "metainflammation" [16]. Thus, we hypothesized that the previously reported anti-inflammatory activity of GKT may influence obesity or its related diseases.

We, therefore, investigated the antioxidant and antiadipogenic effects of GKT. We analyzed its scavenging activities on 2,2'-azinobis-(3-ethylbenzothiazoline-6-sulfonic acid) (ABTS) and 2,2-diphenyl-1-picrylhydrazyl (DPPH) radicals in in vitro systems. The effect on low-density lipoprotein (LDL) oxidation was assessed by measuring production of malondialdehyde (MDA). In addition, its inhibitory effects on adipogenesis, the process by which preadipocytes become differentiated adipocytes [17], were determined by Oil Red $\mathrm{O}$ staining and assays for triglyceride content, 
TABLE 1: Composition of GKT.

\begin{tabular}{|c|c|c|c|c|}
\hline Herbal medicine & Scientific name & Supplier & Source & Amount (g) \\
\hline Puerariae Radix & Pueraria lobata & Omniherb & Jecheon, Korea & 9.0 \\
\hline Cinnamomi Ramulus & Cinnamomi cassia & HMAX & China & 6.0 \\
\hline Ephedrae Herba & Ephedra sinica & HMAX & China & 6.0 \\
\hline Paeoniae Radix & Paeonia lactiflora & Omniherb & Hwasun, Korea & 6.0 \\
\hline Glycyrrhizae Radix et Rhizoma & Glycyrrhiza uralensis & HMAX & China & 6.0 \\
\hline Zingiberis Rhizoma Crudus & Zingiber officinale & Omniherb & Yeongcheon, Korea & 6.0 \\
\hline Zizyphi Fructus & Ziziphus jujuba & Omniherb & Yeongcheon, Korea & 5.0 \\
\hline \multicolumn{2}{|c|}{ Total amount } & & & 44.0 \\
\hline
\end{tabular}

glycerol-3-phosphate dehydrogenase (GPDH) activity, and leptin production in differentiated 3T3-L1 adipocytes.

\section{Materials and Methods}

2.1. Plant Materials. The seven herbal medicines forming GKT were purchased from Omniherb (Yeongcheon, Korea) and HMAX (Jecheon, Korea). The origin of these herbal medicines was taxonomically confirmed by Professor Je Hyun Lee, Dongguk University, Gyeongju, Korea. A voucher specimen (2008-KE02-1 KE02-7) has been deposited at the Herbal Medicine Formulation Research Group, Korea Institute of Oriental Medicine.

2.2. Preparation of GKT Water Extract. A GKT decoction consisting of seven herbal medicines including Puerariae Radix, Cinnamomi Ramulus, Ephedrae Herba, Paeoniae Radix, Glycyrrhizae Radix et Rhizoma, Zingiberis Rhizoma Crudus, and Zizyphi Fructus was mixed (Table 1; $3.5 \mathrm{~kg}$; $44.0 \mathrm{~g} \times 80)$ and extracted in a 10 -fold mass of water at $100^{\circ} \mathrm{C}$ for $2 \mathrm{~h}$ under pressure $\left(1 \mathrm{kgf} / \mathrm{cm}^{2}\right)$ using an electric extractor (COSMOS-660; Kyungseo Machine Co., Incheon, Korea). The water extract was then filtered through a standard sieve (number 270, $53 \mu \mathrm{m}$; Chung Gye Sang Gong Sa, Seoul, Korea) and the solution was evaporated to dryness and freeze-dried to give a powder. The yield of GKT water extract was $12.6 \%$ (441.6 g).

2.3. ABTS Radical Scavenging Activity. The free radical scavenging activity of GKT extract on ABTS was assessed by the method described by Re et al. [18] with slight modifications. ABTS radical cation was produced by reacting $7 \mathrm{mM}$ ABTS solution with $2.45 \mathrm{mM}$ potassium persulfate in the dark at room temperature for $16 \mathrm{~h}$. Absorbance of the reactant was later adjusted to 0.7 at a wavelength of $734 \mathrm{~nm}$. A $100 \mu \mathrm{L}$ aliquot of GKT solution at different concentrations (12.5-

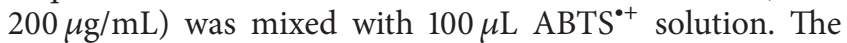
reaction mixture was incubated for $5 \mathrm{~min}$ in the dark at room temperature. The absorbance of the resulting solution was measured at $734 \mathrm{~nm}$ with a spectrophotometer (Benchmark Plus, Bio-Rad, Hercules, CA). The radical scavenging capacity of the tested samples was calculated using the following equation:

Scavenging activity (\%)

$$
=\left(1-\frac{\text { Absorbance of sample }}{\text { Absorbance of control }}\right) \times 100 \text {. }
$$

2.4. DPPH Radical Scavenging Activity. The free radical scavenging activity of GKT extract on DPPH was assessed using the method described by Moreno et al. [19]. In brief, a $100 \mu \mathrm{L}$ aliquot of GKT sample solution at different concentrations $(50-400 \mu \mathrm{g} / \mathrm{mL})$ was mixed with $100 \mu \mathrm{L}$ DPPH solution $(0.15 \mathrm{mM}$ in methanol). The reaction mixture was incubated for $30 \mathrm{~min}$ in the dark at room temperature. The absorbance of the resulting solution was measured at $517 \mathrm{~nm}$ with a spectrophotometer (Benchmark Plus, Bio-Rad, Hercules, CA). The radical scavenging capacity of the tested samples was calculated using the above formula.

2.5. Oxidation of $\mathrm{LDL}$ by $\mathrm{CuSO}_{4}$. LDL samples $(500 \mu \mathrm{g}$ protein/mL, Biomedical Technologies, Stoughton, MA) were prepared at $37^{\circ} \mathrm{C}$ in a medium containing $10 \mathrm{mM}$ phosphate buffer $(\mathrm{pH} 7.4)$ and various concentrations $(15.7-250 \mu \mathrm{g} / \mathrm{mL})$ of GKT extract. After $5 \mathrm{~min}$, oxidation was initiated by the addition of $\mathrm{CuSO}_{4}(25 \mu \mathrm{M})$. After $6 \mathrm{~h}$ of oxidation, lipid peroxidation (Section 2.6) and electrophoretic mobility (Section 2.7) of the LDL were measured as described below.

2.6. Determination of MDA Using TBARS Assay. Lipid peroxidation of LDL was estimated by the determination of the level of MDA using a TBARS assay kit (Bioassay Systems LLC, Hayward, CA) according to the manufacturer's protocols. After oxidation, $50 \mu \mathrm{g}$ of LDLs was mixed with $200 \mu \mathrm{L}$ of thiobarbituric acid (TBA) and incubated at $100^{\circ} \mathrm{C}$ for $30 \mathrm{~min}$. After completing the reaction, the absorbance at $535 \mathrm{~nm}$ was measured using a spectrophotometer.

2.7. Relative Electrophoretic Mobility (REM) Assay. The electrophoretic mobility of LDLs was measured using agarose gel ( $0.8 \%$ agarose in TAE buffer) electrophoresis and Coomassie Brilliant Blue R-250 staining. Electrophoresis was performed at $100 \mathrm{~V}$ for $30 \mathrm{~min}$. REM was defined as the ratio of the distances migrated from the origin by oxidized LDL (oxLDL) versus native LDL.

2.8. Cell Culture and Differentiation. Mouse preadipocyte cell line 3T3-L1 was obtained from the American Type Culture Collection (ATCC, CL-173, Rockville, MD). The cells were cultured in DMEM (Gibco BRL, Carlsbad, CA) supplemented with $10 \%$ newborn calf serum (NCS, Gibco BRL, Carlsbad, CA) at $37^{\circ} \mathrm{C}$. For adipocyte differentiation, the cells were stimulated with 3T3-L1 differentiation medium containing isobutylmethylxanthine, dexamethasone, and insulin 
(MDI) (Zenbio Inc., Research Triangle Park, NC) for $48 \mathrm{~h}$ after reaching confluence. The medium was switched to DMEM containing 10\% fetal bovine serum (FBS) and $1 \mu \mathrm{g} / \mathrm{mL}$ insulin after 2 days and then changed to DMEM containing 10\% FBS for an additional 4 days. Cells were treated with GKT extract $(0,25,50,100,200$, or $400 \mu \mathrm{g} / \mathrm{mL})$ during 8 days of the differentiation period. GW9662 (Sigma, St. Louis, MO), a peroxisome proliferator-activated receptorgamma (PPAR- $\gamma$ ) antagonist, was used as a positive control.

2.9. Cytotoxicity Assay. Undifferentiated 3T3-L1 cells were treated with various concentrations of GKT for $24 \mathrm{~h}$. To obtain differentiated adipocytes, 3T3-L1 preadipocytes were differentiated for 8 days in the presence of GKT. The cells were incubated with GKT extract at $37^{\circ} \mathrm{C}$ under $5 \% \mathrm{CO}_{2}$. Cell counting kit- (CCK-) 8 solution (Dojindo, Kumamoto, Japan) was added and incubated for $4 \mathrm{~h}$. After incubation, the absorbance was read at $450 \mathrm{~nm}$ using a microplate reader (Benchmark Plus, Bio-Rad, Hercules, CA).

2.10. Oil Red O Staining. 3T3-L1 preadipocytes were differentiated into adipocytes by adding isobutylmethylxanthine, dexamethasone, and insulin (MDI) for 8 days. The cells were treated with or without GKT $(200 \mu \mathrm{g} / \mathrm{mL})$ or GW9662 $(20 \mu \mathrm{M})$ during the differentiation period. The differentiated 3T3-L1 cells were fixed with $10 \%$ formalin for $15 \mathrm{~min}$ at room temperature and washed with $70 \%$ ethanol and phosphatebuffered saline (PBS). The cells were stained with Oil Red O (Sigma, St. Louis, MO) for $5 \mathrm{~min}$ and then washed with PBS. Cell images were collected using an Olympus CKX41 inverted microscope (Olympus, Tokyo, Japan). Stained oil droplets were dissolved in isopropyl alcohol and measured by reading the absorbance at $520 \mathrm{~nm}$.

2.11. Triglyceride Quantification Assay. 3T3-L1 preadipocytes were differentiated into adipocytes by adding MDI for 8 days. The cells were treated with or without GKT $(25,50,100,200$, or $400 \mu \mathrm{g} / \mathrm{mL})$ or GW9662 $(20 \mu \mathrm{M})$ during the differentiation period. The triglyceride content of cells was enzymatically measured using a commercial kit (BioVision Inc., Milpitas, CA). Briefly, the 3T3-L1 adipocytes treated with GKT were homogenized in 5\% NP-40 assay buffer and the sample was slowly heated to solubilize all triglycerides. The samples were mixed with lipase and triglyceride reaction mixture. After $1 \mathrm{~h}$ of incubation, the sample absorbance was measured at $570 \mathrm{~nm}$.

2.12. GPDH Activity Assay. After the induction of adipocyte differentiation in the presence of GKT, 3T3-L1 cells were washed twice with PBS. GPDH activity was determined by using a commercial kit (TAKARA, Tokyo, Japan) and monitoring the dihydroxyacetone phosphate-dependent oxidation of nicotinamide adenine dinucleotide (NADH) at $340 \mathrm{~nm}$ and the results were expressed as unit/mg of protein.

2.13. Leptin Immunoassay. Leptin levels were assayed using a mouse leptin immunoassay kit (R\&D Systems, Minneapolis, $\mathrm{MN}$ ) according to the manufacturer's instructions. In brief, the culture supernatant was collected from the differentiated
3T3-L1 with or without GKT treatment. An equal ratio of the supernatants $(50 \mu \mathrm{L})$ and assay diluent $\operatorname{RD} 1 W(50 \mu \mathrm{L})$ was added to wells of a 96-well plate and incubated for $2 \mathrm{~h}$ at room temperature. After washing 5 times with $400 \mu \mathrm{L}$ of wash buffer, $100 \mu \mathrm{L}$ of mouse leptin conjugate was added to each well and incubated for $2 \mathrm{~h}$ at room temperature. After washing 5 times, $100 \mu \mathrm{L}$ of substrate solution was added to each well and incubated for $30 \mathrm{~min}$ at room temperature in the dark. Finally, $100 \mu \mathrm{L}$ of stop solution was added to each well and the absorbance was measured at $450 \mathrm{~nm}$.

2.14. Chemicals and Reagents for HPLC Analysis. Cinnamaldehyde, cinnamic acid, glycyrrhizin, liquiritin, paeoniflorin, and puerarin (all purity $\geq 98.0 \%$ ) were purchased from Wako Fine Chemicals (Osaka, Japan). The HPLC-grade reagents methanol, acetonitrile, and water were obtained from J. T. Baker (Phillipsburg, NJ). Glacial acetic acid was obtained from Junsei Chemical Co. (Tokyo, Japan).

2.15. Preparation of Standard and Sample Solutions for HPLC Analysis. Methanol standard stock solutions were made containing each of cinnamic acid, glycyrrhizin, liquiritin, paeoniflorin, and puerarin at $1.0 \mathrm{mg} / \mathrm{mL}$. Cinnamaldehyde was dissolved in methanol at a concentration of $1.05 \mathrm{mg} / \mathrm{mL}$. The stock solutions were stored below $4^{\circ} \mathrm{C}$.

For HPLC analysis, lyophilized GKT extract was weighed $(200 \mathrm{mg})$ into a $20 \mathrm{~mL}$ flask and distilled water was added to the volumetric mark, and then the mixture was passed through a $0.2 \mu \mathrm{m}$ syringe filter before injection into the HPLC system.

2.16. HPLC Analysis of GKT. HPLC was performed on a Shimadzu LC-20A HPLC system (Shimadzu Co., Kyoto, Japan) consisting of a solvent delivery unit, an on-line degasser, a column oven, an autosampler, and a photodiode array (PDA) detector. The data processor employed LCsolution software (Version 1.24). The analytical column used was a Gemini $\mathrm{C}_{18}$ $(250 \times 4.6 \mathrm{~mm}$; particle size $5 \mu \mathrm{m}$, Phenomenex, Torrance, CA) maintained at $40^{\circ} \mathrm{C}$. The mobile consisted of solvent A $\left(1.0 \%, v / v\right.$, acetic acid in $\left.\mathrm{H}_{2} \mathrm{O}\right)$ and solvent $\mathrm{B}(1.0 \%$, v/v, acetic acid in acetonitrile). The gradient flow was as follows: $5-70 \%$ B for $0-40 \mathrm{~min}, 70-100 \%$ B for $40-45 \mathrm{~min}, 100 \%$ B for $45-50 \mathrm{~min}$, and $100-5 \%$ B for $55 \mathrm{~min}$. The flow rate was $1.0 \mathrm{~mL} / \mathrm{min}$ and the injection volume was $10 \mu \mathrm{L}$. The quantitative analysis of the six compounds was carried out at $230 \mathrm{~nm}$ (paeoniflorin), $254 \mathrm{~nm}$ (glycyrrhizin and puerarin), and $280 \mathrm{~nm}$ (cinnamaldehyde, cinnamic acid, and liquiritin).

2.17. Statistical Analysis. All data were presented as mean \pm standard error of the mean (S.E.M.). Group differences were assessed by one-way ANOVA and post hoc Tukey's multiple comparison test using Graphpad InStat ver.3.10 (Graphpad Software, Inc., San Diego, CA). Significance of differences from the normal control was taken as $P<0.05$.

\section{Results}

3.1. HPLC Analysis of GKT. All calibration curves were obtained by assessment of peak areas from standard 
TABLE 2: Regression data, linear range, correlation coefficient, LOD, and LOQ for marker compounds $(n=3)$.

\begin{tabular}{lccccc}
\hline Compound & Linear range $(\mu \mathrm{g} / \mathrm{mL})$ & Regression equation $^{\mathrm{a}}$ & ${\text { Correlation coefficient }\left(R^{2}\right)}^{\mathrm{LOD}^{\mathrm{b}}(\mu \mathrm{g} / \mathrm{mL})}$ & $\mathrm{LOQ}^{\mathrm{c}}(\mu \mathrm{g} / \mathrm{mL})$ \\
\hline Puerarin & $2.34-300.00$ & $y=39814.06 x+35976.26$ & 1.0000 & 0.04 & 0.15 \\
Paeoniflorin & $0.78-100.00$ & $y=38738.30 x-15273.19$ & 0.9999 & 0.05 & 0.15 \\
Liquiritin & $1.56-200.00$ & $y=18495.04 x+12062.72$ & 1.0000 & 0.03 & 0.11 \\
Cinnamic acid & $2.34-300.00$ & $y=27338.38 x+31461.88$ & 1.0000 & 0.02 & 0.01 \\
Cinnamaldehyde & $1.64-105.00$ & $y=122000.31 x+62587.19$ & 1.0000 & 0.03 \\
Glycyrrhizin & $2.34-300.00$ & $y=8266.43 x+5843.51$ & 1.0000 & 0.36 \\
\hline
\end{tabular}

a $y$ : peak area (mAU) of compounds; $x$ : concentration $(\mu \mathrm{g} / \mathrm{mL})$ of compounds.

${ }^{\mathrm{b}} \mathrm{LOD}=3 \times$ signal-to-noise ratio.

${ }^{\mathrm{c}} \mathrm{LOQ}=10 \times$ signal-to-noise ratio.

TABLE 3: Contents of six compounds in the GKT by HPLC $(n=3)$.

\begin{tabular}{lcccc}
\hline Compound & Mean $(\mathrm{mg} / \mathrm{g})$ & SD & RSD $(\%)$ & Source \\
\hline Puerarin & 10.29 & 0.02 & 0.21 & Puerariae Radix \\
Paeoniflorin & 3.55 & 0.02 & 0.69 & Paeoniae Radix \\
Liquiritin & 6.64 & 0.05 & 0.75 & 2.44 \\
Cinnamic acid & 2.01 & 0.05 & 0.50 & Glycyrrhizae Radix et Rhizoma \\
Cinnamaldehyde & 7.30 & 0.04 & 0.76 & Cinnamomi Ramulus \\
Glycyrrhizin & 12.17 & 0.09 & & Cinnamomi Ramulus \\
\end{tabular}

solutions in the concentration ranges: puerarin, cinnamic acid, and glycyrrhizin, $2.34-300.00 \mu \mathrm{g} / \mathrm{mL}$; paeoniflorin, liquiritin, and cinnamaldehyde, $0.78-100.00 \mu \mathrm{g} / \mathrm{mL}, 1.56-$ $200.00 \mu \mathrm{g} / \mathrm{mL}$, and $1.64-105.00 \mu \mathrm{g} / \mathrm{mL}$, respectively. The retention times were 13.81, 15.72, 17.65, 25.77, 28.08, and $33.55 \mathrm{~min}$ for puerarin, paeoniflorin, liquiritin, cinnamic acid, cinnamaldehyde, and glycyrrhizin, respectively. The calibration curves, correlation coefficients $\left(R^{2}\right)$, limit of detection (LOD), and limit of quantification (LOQ) of the six marker compounds are summarized in Table 2. Using optimized chromatography conditions, a three-dimensional chromatogram was obtained using the HPLC-PDA detector (Figure 1). The concentrations of the six marker compounds were $2.01-12.17 \mathrm{mg} / \mathrm{g}$ and are summarized in Table 3.

3.2. Antioxidant Activity of GKT. To evaluate the antioxidant activity of GKT, we tested its scavenging activities on ABTS and DPPH radicals. The ABTS radical scavenging activity of GKT is presented in Table 4. The extracts of GKT showed a dose-dependent radical scavenging activity. The concentration of GKT required for $50 \%$ inhibition $\left(\mathrm{IC}_{50}\right)$ of ABTS radicals was $51.16 \mu \mathrm{g} / \mathrm{mL}$, while the $\mathrm{IC}_{50}$ value of ascorbic acid, as a positive control, was $3.22 \mu \mathrm{g} / \mathrm{mL}$. The antioxidant activities obtained for GKT by the DPPH method are shown in Table 5. Similar to the ABTS assay, GKT reduced DPPH radical formation in a concentration-dependent manner. The $\mathrm{IC}_{50}$ of GKT against DPPH radicals was $242.96 \mu \mathrm{g} / \mathrm{mL}$, while the $\mathrm{IC}_{50}$ value of ascorbic acid was $10.43 \mu \mathrm{g} / \mathrm{mL}$.

3.3. Effect of GKT on $\mathrm{Cu}^{2+}$-Mediated Oxidation of LDL. The generation of MDA equivalents during LDL oxidation was estimated by the TBARS assay. As shown in Figure 2(a), when LDL was incubated with $\mathrm{CuSO}_{4}$ for $6 \mathrm{~h}$, a significant
TABLE 4: Scavenging effects of GKT on $\mathrm{ABTS}^{*+}$.

\begin{tabular}{lccc}
\hline Sample & $\begin{array}{c}\text { Concentration } \\
(\mu \mathrm{g} / \mathrm{mL})\end{array}$ & $\begin{array}{c}\text { Scavenging effect } \\
(\%)\end{array}$ & $\begin{array}{c}\mathrm{IC}_{50}{ }^{1} \\
(\mu \mathrm{g} / \mathrm{mL})\end{array}$ \\
\hline & 12.5 & $19.24 \pm 1.19$ & \\
$\mathrm{GKT}^{2}$ & 25 & $32.85 \pm 2.80$ & \\
& 50 & $58.08 \pm 3.51$ & $51.16 \pm 2.67$ \\
& 100 & $82.35 \pm 0.26$ & \\
& 200 & $97.46 \pm 0.30$ & \\
$\mathrm{AA}^{3}$ & 1.25 & $21.15 \pm 0.19$ & \\
& 2.5 & $40.61 \pm 1.44$ & $3.22 \pm 0.06$ \\
\hline
\end{tabular}

${ }^{1}$ Concentration required for $50 \%$ reduction of $\mathrm{ABTS}^{\circ+}$ at 5 min reaction.

${ }^{2}$ Galkeun-tang and ${ }^{3}$ ascorbic acid.

Each value is the mean \pm S.E.M. of triplicate determinations.

TABLE 5: Scavenging effects of GKT on DPPH.

\begin{tabular}{lccc}
\hline Sample & $\begin{array}{c}\text { Concentration } \\
(\mu \mathrm{g} / \mathrm{mL})\end{array}$ & $\begin{array}{c}\text { Scavenging effect } \\
(\%)\end{array}$ & $\begin{array}{c}\mathrm{IC}_{50}{ }^{1} \\
(\mu \mathrm{g} / \mathrm{mL})\end{array}$ \\
\hline & 50 & $6.33 \pm 0.14$ & \\
$\mathrm{GKT}^{2}$ & 100 & $22.64 \pm 0.49$ & $242.96 \pm 2.66$ \\
& 200 & $47.66 \pm 1.07$ & \\
& 400 & $78.70 \pm 0.60$ & \\
$\mathrm{AA}^{3}$ & 1.25 & $21.15 \pm 0.19$ & \\
& 2.5 & $40.61 \pm 1.44$ & $3.22 \pm 0.06$ \\
\hline
\end{tabular}

${ }^{1}$ Concentration required for $50 \%$ reduction of DPPH at 30 min reaction.

${ }^{2}$ Galgeun-tang and ${ }^{3}$ ascorbic acid.

Each value is the mean \pm S.E.M. of triplicate determinations. 


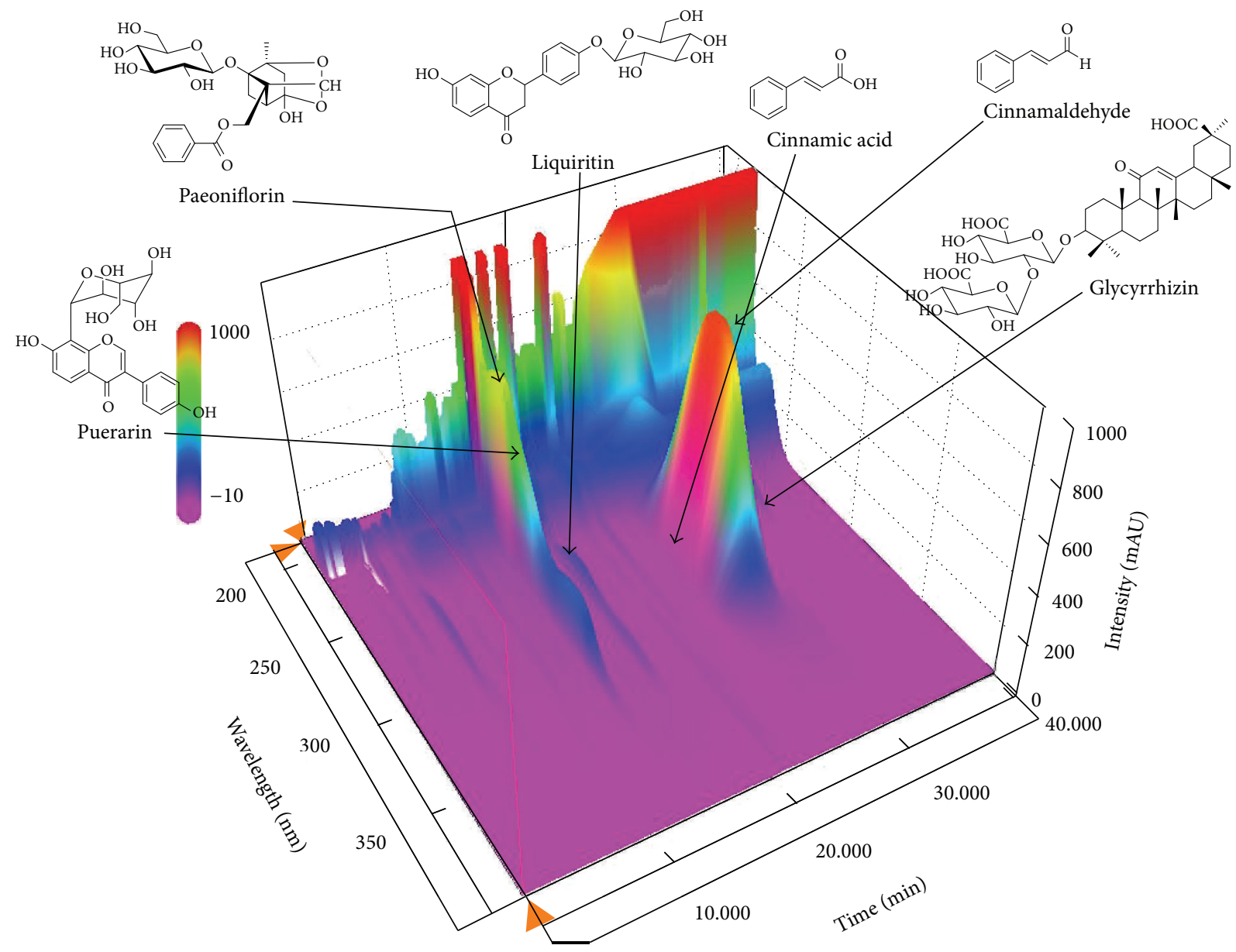

FIGURE 1: Three-dimensional chromatogram of GKT by HPLC-PDA.

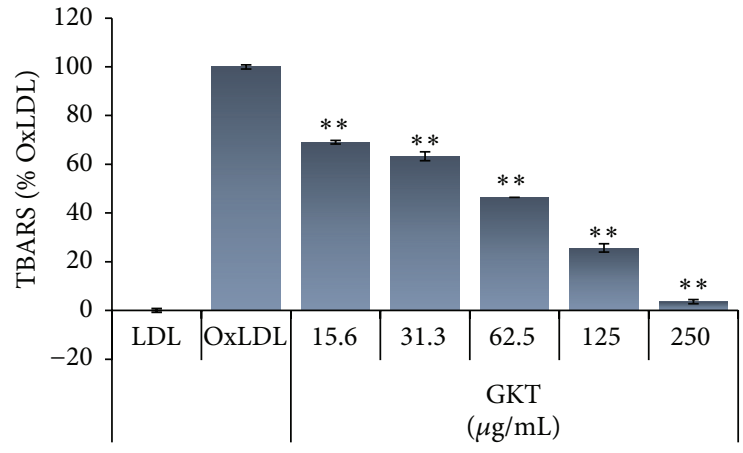

(a)

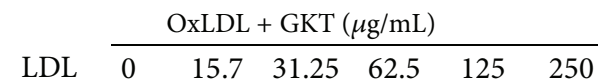
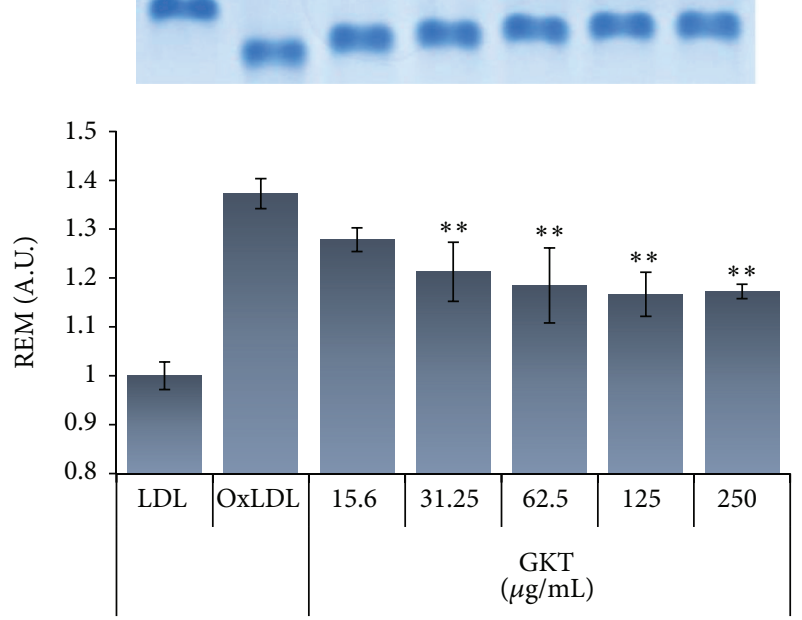

(b)

FIgURE 2: Inhibitory effects of GKT on $\mathrm{Cu}^{2+}$-induced LDL oxidation. The indicated concentrations of GKT and LDLs were incubated with $\mathrm{CuSO}_{4}$ for $6 \mathrm{~h}$ at $37^{\circ} \mathrm{C}$. The TBARS level (a) and electrophoretic mobility (b) of LDLs were measured using a TBARS assay kit and agarose gel electrophoresis, respectively. The data are presented as the mean values of three experiments' \pm S.E.M. ${ }^{* *} P<0.01$ compared with the oxLDL group. 


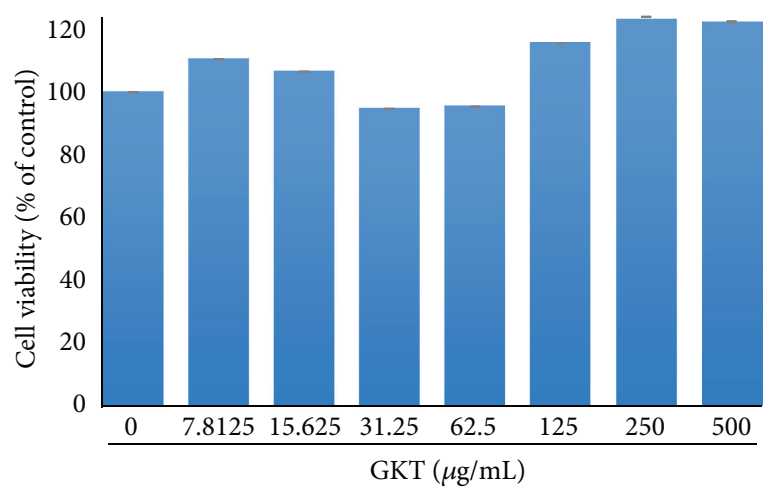

(a)

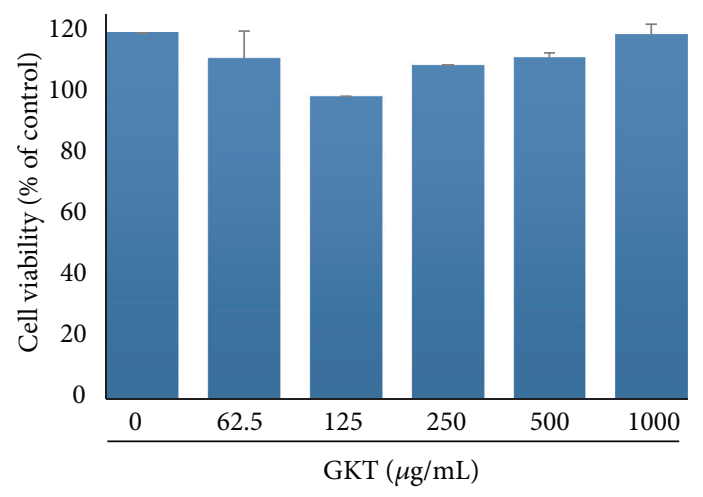

(b)

FIGURE 3: Cytotoxic effects of GKT extract in 3T3-L1 cells. (a) Undifferentiated 3T3-L1 cells were treated with various concentrations of GKT $(0,7.8125,15.625,31.25,62.5,125,250$, or $500 \mu \mathrm{g} / \mathrm{mL})$ for $24 \mathrm{~h}$. (b) Adipocyte differentiation was induced by adding isobutylmethylxanthine, dexamethasone, and insulin (MDI) to 3T3-L1 preadipocytes for 8 days. The cells were exposed to various concentrations of GKT (0, 62.5, $125,250,500$, or $1000 \mu \mathrm{g} / \mathrm{mL}$ ) during the differentiation period. Cell viability was determined using a CCK- 8 assay kit by measuring the absorbance at $450 \mathrm{~nm}$. Data are presented as the mean \pm S.E.M.

increase in TBARS was detected. In contrast, GKT significantly reduced the amount of TBARS formed in a dosedependent manner $\left(\mathrm{IC}_{50}: 53.23 \mu \mathrm{g} / \mathrm{mL}\right)$. The alteration of mobility in agarose gel electrophoresis reflects the increase in the negative charge of LDL particles which occurs during oxidation [20]. When the oxidation was carried out in the presence of GKT, the increase in electrophoretic mobility of oxLDL was significantly reduced (Figure 2(b)). These data suggest that GKT has an inhibitory effect on LDL oxidation.

3.4. Cytotoxic Effects of GKT in 3T3-L1 Cells. To evaluate the possible cytotoxicity of GKT against 3T3-L1 preadipocytes, the cells were treated with various concentrations of GKT for $24 \mathrm{~h}$. As shown in Figure 3(a), GKT had no cytotoxicity against 3T3-L1 preadipocytes. Additionally, the cytotoxicity of GKT was assessed in the differentiated adipocytes. During differentiation for 8 days, the cells were exposed to various concentrations of GKT. No significant cytotoxic effect was observed in GKT-treated adipocytes (Figure 3(b)).

\subsection{The Inhibitory Effects of GKT on Adipogenesis of 3T3-L1} Adipocytes. Triglyceride production is one of the important events which occurs during adipogenesis [21]. Oil Red O staining was carried out to examine lipid accumulation in the differentiated 3T3-L1 adipocytes. The number of lipid droplets detectable with Oil Red O staining was obviously increased in adipocytes compared with preadipocytes (Figure 4(a)). However, GKT treatment significantly reduced lipid accumulation compared with the untreated differentiated cells. In addition, the intracellular triglyceride content was measured in 3T3-L1 adipocytes treated with GKT. Consistent with the results of Oil Red O staining, triglyceride production was significantly increased in the differentiated adipocytes compared with preadipocytes, but GKT significantly inhibited triglyceride production compared with untreated differentiated cells (Figure 4(b)).
GPDH is an enzyme that generates glycerol-3-phosphate from dihydroxyacetone phosphate for lipid biosynthesis in adipocytes [22]. As shown in Figure 5(a), treatment with GKT at the concentration of $25 \sim 400 \mu \mathrm{g} / \mathrm{mL}$ significantly inhibited the activity of GPDH compared with untreated differentiated adipocytes. Consistent with this, GKT also had a suppressive effect on secretion of leptin, a key adipokine [23], compared with the untreated differentiated cells (Figure 5(b)). Similarly, treatment with GW9662, the positive control, dramatically inhibited adipogenesis in 3T3L1 cells.

\section{Discussion}

In the present study, we demonstrate that a traditional herbal medicine GKT has antioxidant and antiadipogenesis properties. For quality control of the GKT extract, we performed a quantitative determination of the six main components in GKT using HPLC coupled with a PDA. The investigated components were as follows: puerarin form Puerariae Radix, cinnamaldehyde and cinnamic acid from Cinnamomi Ramulus, paeoniflorin from Paeoniae Radix, and liquiritin and glycyrrhizin from Glycyrrhizae Radix et Rhizoma. The optimized HPLC-PDA method was applied for simultaneous quantitation of the six components in GKT (Figure 1). Among these components, puerarin and glycyrrhizin, which are marker components of Puerariae Radix and Glycyrrhizae Radix et Rhizoma, were detected at $10.29 \mathrm{mg} / \mathrm{g}$ and $12.17 \mathrm{mg} / \mathrm{g}$, respectively, as the major components of GKT (Table 3). The establishment of this HPLC-PDA method will be helpful in improving quality control of GKT.

In in vitro assay systems, GKT showed significant scavenging effects against ABTS and DPPH radicals (Table 4), and it inhibited LDL oxidation mediated by $\mathrm{CuSO}_{4}$ in a dosedependent manner (Figure 2). In addition, GKT revealed antiadipogenic activity by blocking lipid accumulation, 

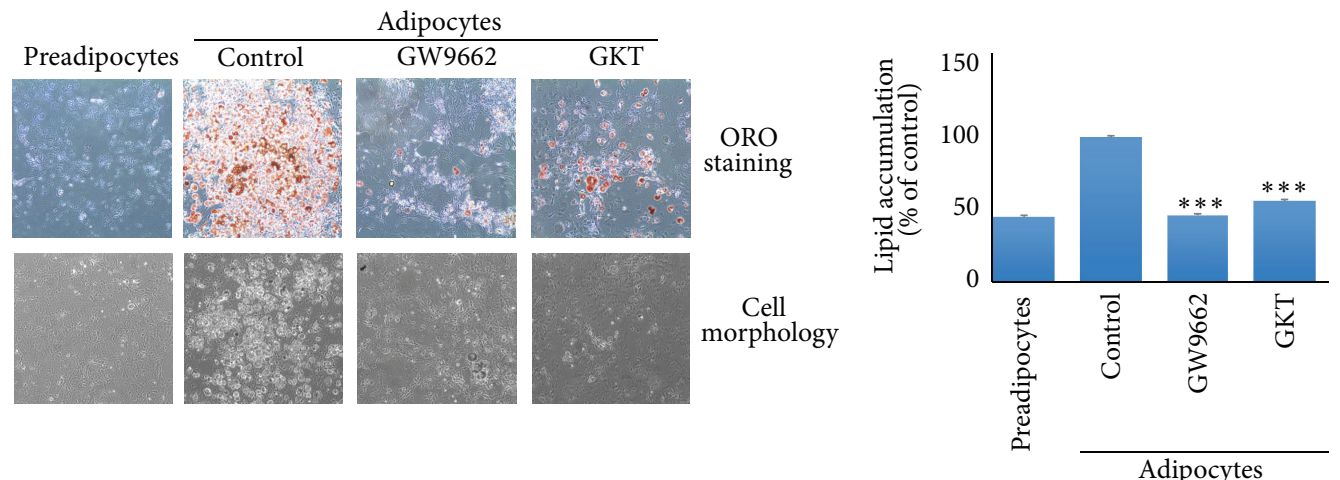

(a)

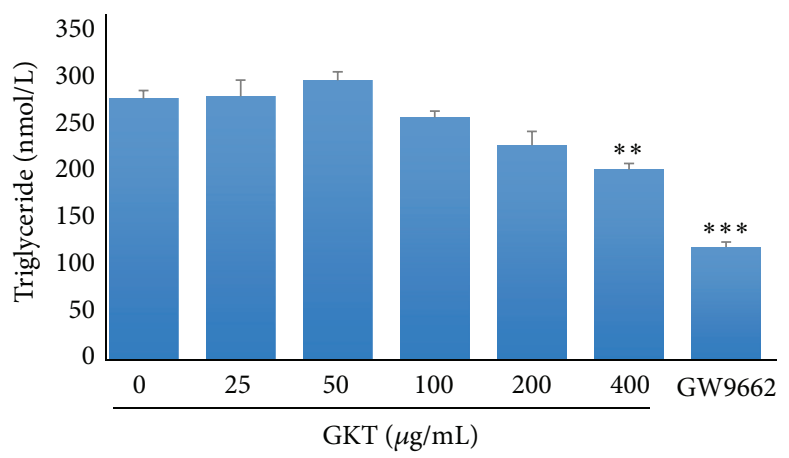

(b)

FIGURE 4: Inhibitory effect of GKT extract on triglyceride accumulation in 3T3-L1 adipocytes. 3T3-L1 preadipocytes were differentiated into adipocytes by adding isobutylmethylxanthine, dexamethasone, and insulin (MDI) for 8 days. The cells were treated with or without GKT or GW9662 $(20 \mu \mathrm{M})$ during the differentiation period. (a and b) Lipid accumulation in the cells was analyzed by Oil Red O staining. (a) The cells stained with Oil Red O were visualized using an Olympus CKX41 inverted microscope at $\times 200$ magnification (left panel). Stained oil droplets were dissolved in isopropyl alcohol and measured by reading absorbance at $520 \mathrm{~nm}$ (right panel). (b) The content of triglyceride was enzymatically measured at $570 \mathrm{~nm}$ using a commercial kit (BioVision Inc., Milpitas, CA). Data are presented as the mean \pm S.E.M. ${ }^{* *} P<0.01$ and ${ }^{* * *} P<0.001$ compared with the differentiated control.

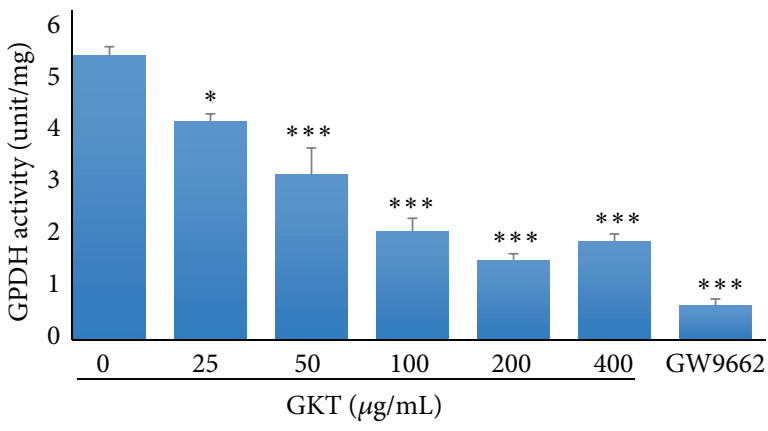

(a)

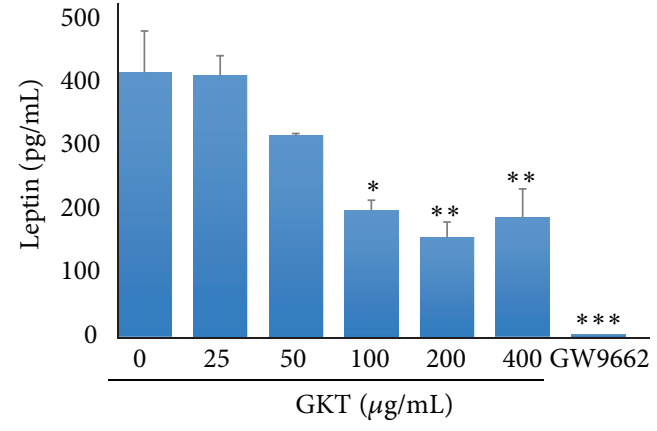

(b)

FIGURE 5: Inhibitory effects of GKT on adipogenesis-related factors in 3T3-L1 adipocytes. 3T3-L1 preadipocytes were differentiated into adipocytes by adding isobutylmethylxanthine, dexamethasone, and insulin (MDI) for 8 days. The cells were exposed to various concentrations of GKT during the differentiation period. (a) GPDH activity of the cells was assessed by measuring the decrease in NADH at $340 \mathrm{~nm}$ using the TAKARA GPDH activity assay kit. (b) Culture supernatant was collected from the GKT-treated cells. Leptin production was determined by ELISA at $540 \mathrm{~nm}$ subtracted from $450 \mathrm{~nm}$ using a mouse leptin immunoassay kit (R\&D Systems). Data are presented as the mean \pm S.E.M. ${ }^{* * *} P<0.001,{ }^{* *} P<0.01$, and ${ }^{*} P<0.05$ compared with the differentiated control. 
triglyceride production, GPDH activity, and leptin production in adipocytes without cytotoxic effects (Figures 3-5).

Oxidative stress plays an important role in the pathogenesis of various diseases including obesity. Thus, the "oxidative stress paradigm" is an appealing concept for developing novel therapeutics [24]. Excess oxidative stress in obese patients coincides with fat accumulation in adipocytes [4]. We induced the cellular differentiation of 3T3-L1 mouse preadipocytes into adipocytes. This cell line is useful for studying adipogenesis [25]. After adipocyte differentiation, a significant increase of intracellular lipid accumulation compared with untreated preadipocytes was observed by Oil Red O staining. In contrast, GKT significantly inhibited the amount of lipid accumulation compared with untreated differentiated adipocytes (Figure 4(a)). Microscopy further confirmed the inhibitory effect of GKT on adipogenesis. GKT treatment markedly reduced the increase in the number and size of adipocytes, a hallmark of adipocyte endocrine function [26]. Lipid droplets consist of a core of lipid esters and a surface lined with a phospholipid monolayer, and, in adipocytes, the lipid ester core contains triglycerides [2729]. Consistent with the results of Oil Red O staining, GKT significantly decreased the content of triglyceride in adipocytes (Figure 4(b)). Furthermore, we evaluated the GPDH activity in differentiated 3T3-L1 adipocytes. GPDH is activated strongly in mature adipocytes and plays a role in the triglyceride biosynthesis pathway [30, 31]. GPDH enzyme activity was significantly decreased in GKT-treated adipocytes (Figure 5(a)). The level of another key factor in adipogenesis, leptin, was measured in adipocytes differentiated in the absence or presence of GKT. Leptin is an adipokine exclusively produced by adipocytes in proportion to triglyceride accumulation [32]. As expected, GKT significantly inhibited the level of secretion of leptin in differentiated 3T3-L1 adipocytes (Figure 5(b)). Similar to our study, several groups have reported dual effects of natural products, including purple sweet potato [33], safflower seed [34], and buckwheat sprout [35], on oxidative stress and adipogenesis. Together, these results suggest the potential of natural products, including herbal medicines, as adipogenesis-preventing substances with antioxidant properties.

Interestingly, several herbal components of GKT have been reported to have antioxidant properties or antiobesity effects. Pueraria lobata extract ameliorated impaired glucose and lipid metabolism in obese mice [36]. Pueraria lobata extract also inhibited tert-butyl hydroperoxideinduced ROS generation [37]. An extract of Cinnamomum cassia twigs inhibited adipocyte differentiation via activation of the insulin signaling pathway in 3T3-L1 preadipocytes [38]. Ephedra sinica extract exerted antioxidant effects by accelerating free radical scavenging activity and oxidant reducing power [39]. These data could support antiadipogenic/antioxidant activities of GKT. Additional studies will be required to confirm the antioxidant/antiobesity effects of GKT using a high fat diet-fed obese animal model.

\section{Conflict of Interests}

The authors have declared that no conflict of interests exists.

\section{Acknowledgment}

This work was supported by a Grant from the Korean Institute of Oriental Medicine (no. K14030).

\section{References}

[1] D. J. Betteridge, "What is oxidative stress?" Metabolism: Clinical and Experimental, vol. 49, no. 2, supplement 1, pp. 3-8, 2000.

[2] M. Valko, D. Leibfritz, J. Moncol, M. T. D. Cronin, M. Mazur, and J. Telser, "Free radicals and antioxidants in normal physiological functions and human disease," International Journal of Biochemistry and Cell Biology, vol. 39, no. 1, pp. 44-84, 2007.

[3] N. Rasouli, B. Molavi, S. C. Elbein, and P. A. Kern, "Ectopic fat accumulation and metabolic syndrome," Diabetes, Obesity and Metabolism, vol. 9, no. 1, pp. 1-10, 2007.

[4] S. Furukawa, T. Fujita, M. Shimabukuro et al., "Increased oxidative stress in obesity and its impact on metabolic syndrome," The Journal of Clinical Investigation, vol. 114, no. 12, pp. 1752-1761, 2004.

[5] C. K. Roberts, R. J. Barnard, R. K. Sindhu, M. Jurczak, A. Ehdaie, and N. D. Vaziri, "Oxidative stress and dysregulation of $\mathrm{NAD}(\mathrm{P}) \mathrm{H}$ oxidase and antioxidant enzymes in diet-induced metabolic syndrome," Metabolism, vol. 55, no. 7, pp. 928-934, 2006.

[6] E. Tumova, W. Sun, P. H. Jones, M. Vrablik, C. M. Ballantyne, and R. C. Hoogeveen, "The impact of rapid weight loss on oxidative stress markers and the expression of the metabolic syndrome in obese individuals," Journal of Obesity, vol. 2013, Article ID 729515, 10 pages, 2013.

[7] I. D. Capel and H. M. Dorrell, "Abnormal antioxidant defence in some tissues of congenitally obese mice," Biochemical Journal, vol. 219, no. 1, pp. 41-49, 1984.

[8] M. Ozata, M. Mergen, C. Oktenli et al., "Increased oxidative stress and hypozincemia in male obesity," Clinical Biochemistry, vol. 35, no. 8, pp. 627-631, 2002.

[9] T. Xue and R. Roy, "Studying traditional Chinese medicine," Science, vol. 300, no. 5620, pp. 740-741, 2003.

[10] D. Normile, "The new face of traditional Chinese medicine," Science, vol. 299, no. 5604, pp. 188-190, 2003.

[11] M. Kurokawa, M. Tsurita, J. Brown, Y. Fukuda, and K. Shiraki, "Effect of interleukin-12 level augmented by Kakkon-to, a herbal medicine, on the early stage of influenza infection in mice," Antiviral Research, vol. 56, no. 2, pp. 183-188, 2002.

[12] M. S. Wu, H. R. Yen, C. W. Chang et al., "Mechanism of action of the suppression of influenza virus replication by Ko-Ken Tang through inhibition of the phosphatidylinositol 3-kinase/Akt signaling pathway and viral RNP nuclear export," Journal of Ethnopharmacology, vol. 134, no. 3, pp. 614-623, 2011.

[13] K. Nagasaka, M. Kurokawa, M. Imakita, K. Terasawa, and K. Shiraki, "Efficacy of Kakkon-to, a traditional herb medicine, in herpes simplex virus type 1 infection in mice," Journal of Medical Virology, vol. 46, no. 1, pp. 28-34, 1995.

[14] K. Muraoka, S. Yoshida, K. Hasegawa et al., "A pharmacologic study on the mechanism of action of Kakkon-to: body temperature elevation and phagocytic activation of macrophages in dogs," Journal of Alternative and Complementary Medicine, vol. 10, no. 5, pp. 841-849, 2004.

[15] H. Yano, S. Hiraki, and S. Hayasaka, "Effects of Kakkon-to and Sairei-to on experimental elevation of aqueous flare in pigmented rabbits," Japanese Journal of Ophthalmology, vol. 43, no. 4, pp. 279-284, 1999. 
[16] G. S. Hotamisligil, "Inflammation and metabolic disorders," Nature, vol. 444, no. 7121, pp. 860-867, 2006.

[17] M. I. Lefterova and M. A. Lazar, "New developments in adipogenesis," Trends in Endocrinology \& Metabolism, vol. 20, no. 3, pp. 107-114, 2009.

[18] R. Re, N. Pellegrini, A. Proteggente, A. Pannala, M. Yang, and C. Rice-Evans, "Antioxidant activity applying an improved ABTS radical cation decolorization assay," Free Radical Biology and Medicine, vol. 26, no. 9-10, pp. 1231-1237, 1999.

[19] M. I. N. Moreno, M. I. Isla, A. R. Sampietro, and M. A. Vattuone, "Comparison of the free radical-scavenging activity of propolis from several regions of Argentina," Journal of Ethnopharmacology, vol. 71, no. 1-2, pp. 109-114, 2000.

[20] D. L. Sparks and M. C. Phillips, "Quantitative measurement of lipoprotein surface charge by agarose gel electrophoresis," Journal of Lipid Research, vol. 33, no. 1, pp. 123-130, 1992.

[21] D. W. Haslam and W. P. T. James, "Obesity," The Lancet, vol. 366, no. 9492, pp. 1197-1209, 2005.

[22] L. P. Kozak and J. T. Jensen, "Genetic and developmental control of multiple forms of L-glycerol 3-phosphate dehydrogenase," The Journal of Biological Chemistry, vol. 249, no. 24, pp. 77757781, 1974.

[23] F. Zhang, M. B. Basinski, J. M. Beals et al., "Crystal structure of the obese protein leptin-E100," Nature, vol. 387, no. 6629, pp. 206-209, 1997.

[24] H. Sies, "Oxidative stress: oxidants and antioxidants," Experimental Physiology, vol. 82, no. 2, pp. 291-295, 1997.

[25] H. Green and M. Meuth, "An established pre-adipose cell line and its differentiation in culture," Cell, vol. 3, no. 2, pp. 127-133, 1974.

[26] T. Tsuda, F. Horio, K. Uchida, H. Aoki, and T. Osawa, "Dietary cyanidin 3-O- $\beta$-D-glucoside-rich purple corn color prevents obesity and ameliorates hyperglycemia in mice," Journal of Nutrition, vol. 133, no. 7, pp. 2125-2130, 2003.

[27] D. J. Murphy and J. Vance, "Mechanisms of lipid-body formation," Trends in Biochemical Sciences, vol. 24, no. 3, pp. 109-115, 1999.

[28] K. Tauchi-Sato, S. Ozeki, T. Houjou, R. Taguchi, and T. Fujimoto, "The surface of lipid droplets is a phospholipid monolayer with a unique fatty acid composition," The Journal of Biological Chemistry, vol. 277, no. 46, pp. 44507-44512, 2002.

[29] T. Fujimoto, Y. Ohsaki, J. Cheng, M. Suzuki, and Y. Shinohara, "Lipid droplets: a classic organelle with new outfits," Histochemistry and Cell Biology, vol. 130, no. 2, pp. 263-279, 2008.

[30] J. Pairault and H. Green, "A study of the adipose conversion of suspended 3T3 cells by using glycerophosphate dehydrogenase as differentiation marker," Proceedings of the National Academy of Sciences of the United States of America, vol. 76, no. 10, pp. 5138-5142, 1979.

[31] L. S. Wise and H. Green, "Participation of one isozyme of cytosolic glycerophosphate dehydrogenase in the adipose conversion of 3T3 cells," The Journal of Biological Chemistry, vol. 254, no. 2, pp. 273-275, 1979.

[32] H. C. Chen and R. V. Farese Jr., "Determination of adipocyte size by computer image analysis," Journal of Lipid Research, vol. 43, no. 6, pp. 986-989, 2002.

[33] J.-H. Ju, H.-S. Yoon, H.-J. Park et al., "Anti-obesity and antioxidative effects of purple sweet potato extract in 3T3-L1 adipocytes in vitro," Journal of Medicinal Food, vol. 14, no. 10, pp. 1097-1106, 2011.
[34] S.-Y. Yu, Y.-J. Lee, J.-D. Kim et al., "Phenolic composition, antioxidant activity and anti-adipogenic effect of hot water extract from safflower (Carthamus tinctorius L.) seed," Nutrients, vol. 5, no. 12, pp. 4894-4907, 2013.

[35] Y.-J. Lee, K.-J. Kim, K.-J. Park, B.-R. Yoon, J.-H. Lim, and O.H. Lee, "Buckwheat (Fagopyrum esculentum M.) sprout treated with methyl jasmonate (MeJA) improved anti-adipogenic activity associated with the oxidative stress system in 3T3-L1 adipocytes," International Journal of Molecular Sciences, vol. 14, no. 1, pp. 1428-1442, 2013.

[36] J. K. Prasain, N. Peng, R. Rajbhandari, and J. M. Wyss, "The Chinese Pueraria root extract (Pueraria lobata) ameliorates impaired glucose and lipid metabolism in obese mice," Phytomedicine, vol. 20, no. 1, pp. 17-23, 2012.

[37] S. E. Jin, Y. K. Son, B.-S. Min, H. A. Jung, and J. S. Choi, "Antiinflammatory and antioxidant activities of constituents isolated from Pueraria lobata roots," Archives of Pharmacal Research, vol. 35, no. 5, pp. 823-837, 2012.

[38] Y. Han, H. W. Jung, H. S. Bae, J.-S. Kang, and Y.-K. Park, "The extract of Cinnamomum cassia twigs inhibits adipocyte differentiation via activation of the insulin signaling pathway in 3T3-L1 preadipocytes," Pharmaceutical Biology, vol. 51, no. 8, pp. 961-967, 2013.

[39] F. L. Song, R. Y. Gan, Y. Zhang, Q. Xiao, L. Kuang, and H. B. Li, "Total phenolic contents and antioxidant capacities of selected chinese medicinal plants," International Journal of Molecular Sciences, vol. 11, no. 6, pp. 2362-2372, 2010. 


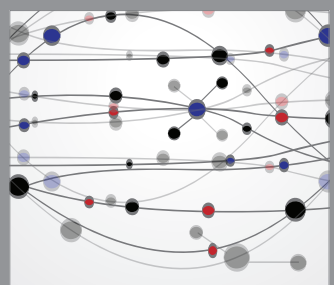

The Scientific World Journal
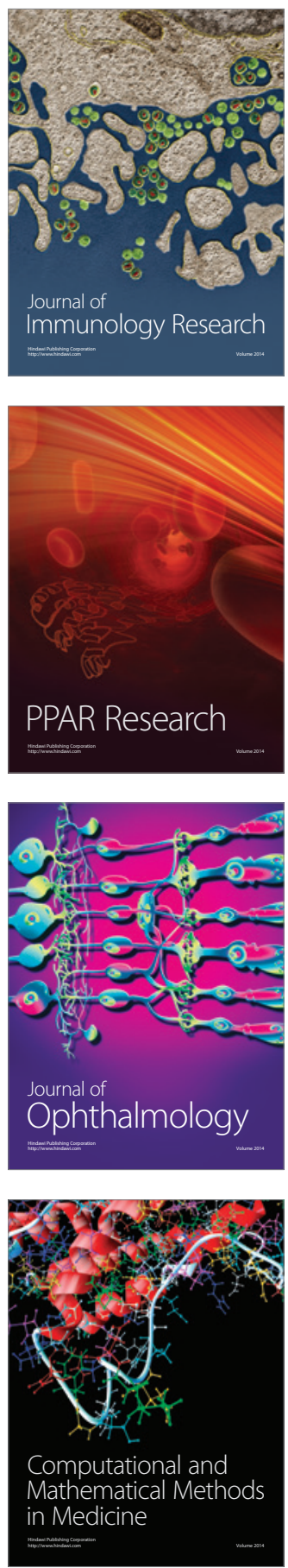

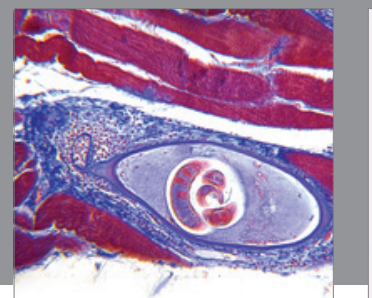

Gastroenterology

Research and Practice
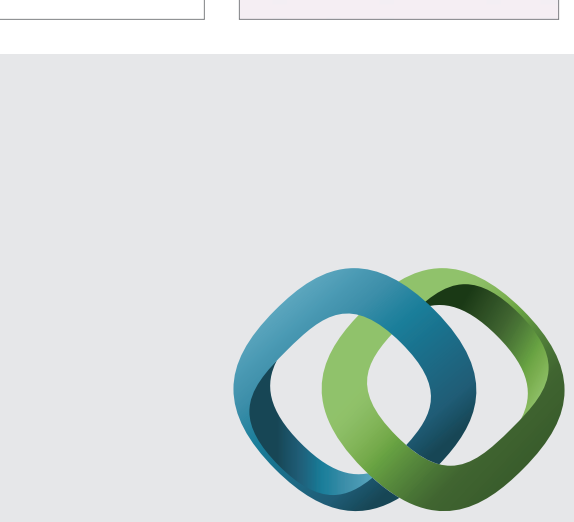

\section{Hindawi}

Submit your manuscripts at

http://www.hindawi.com
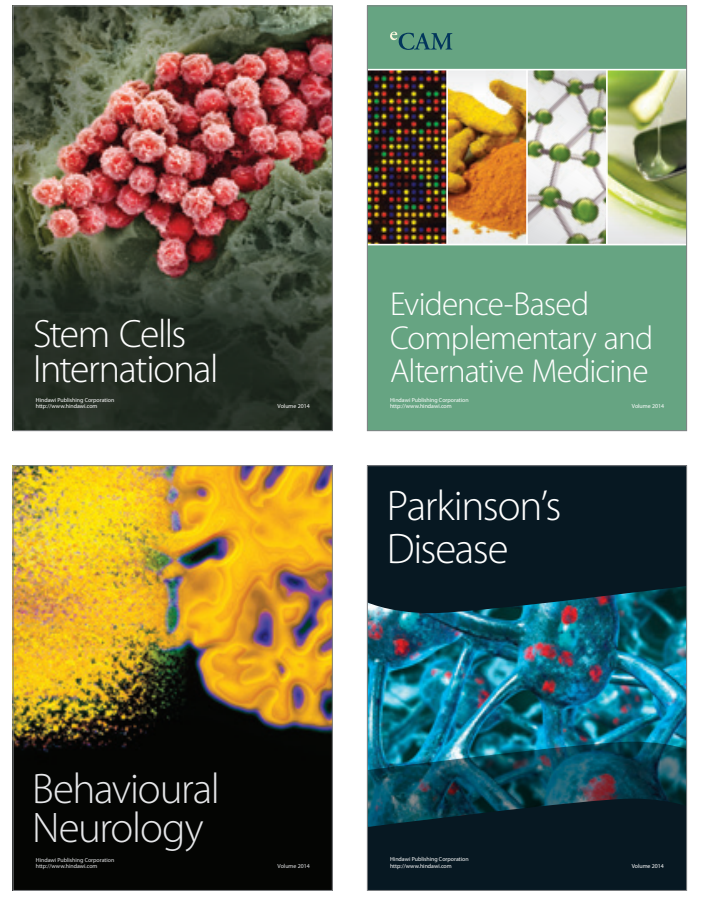
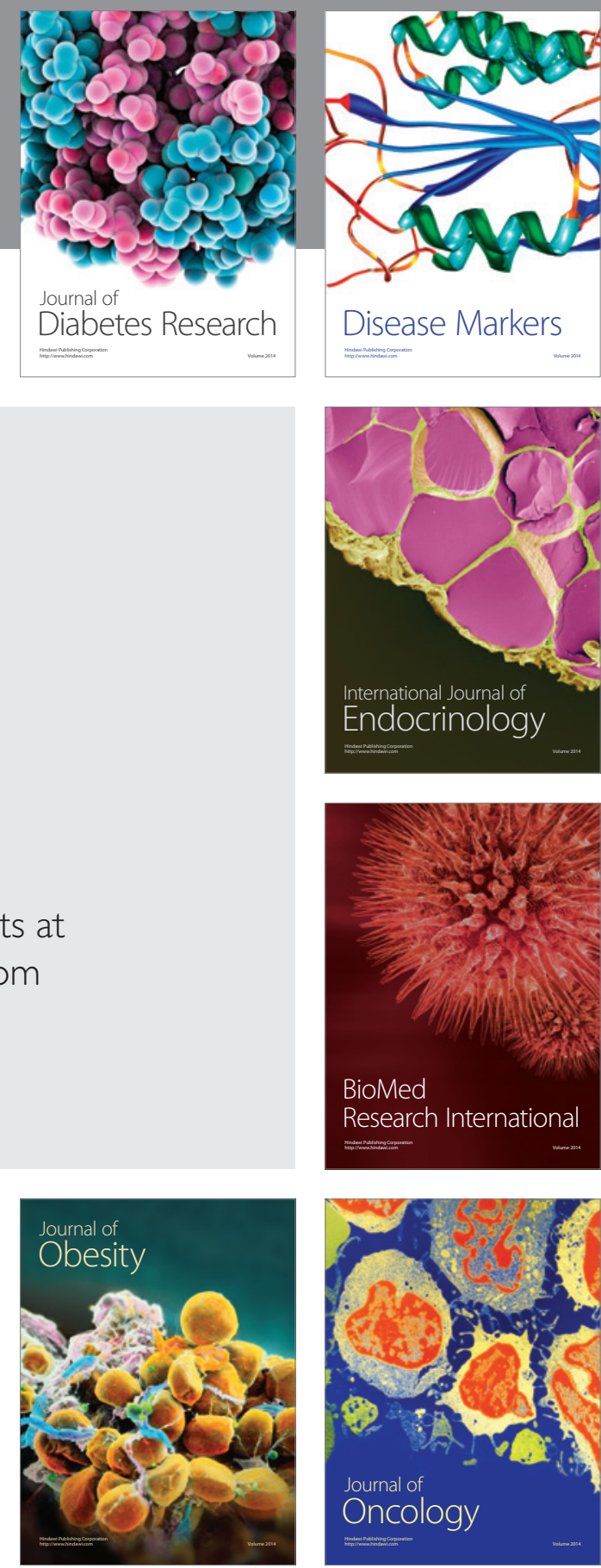

Disease Markers
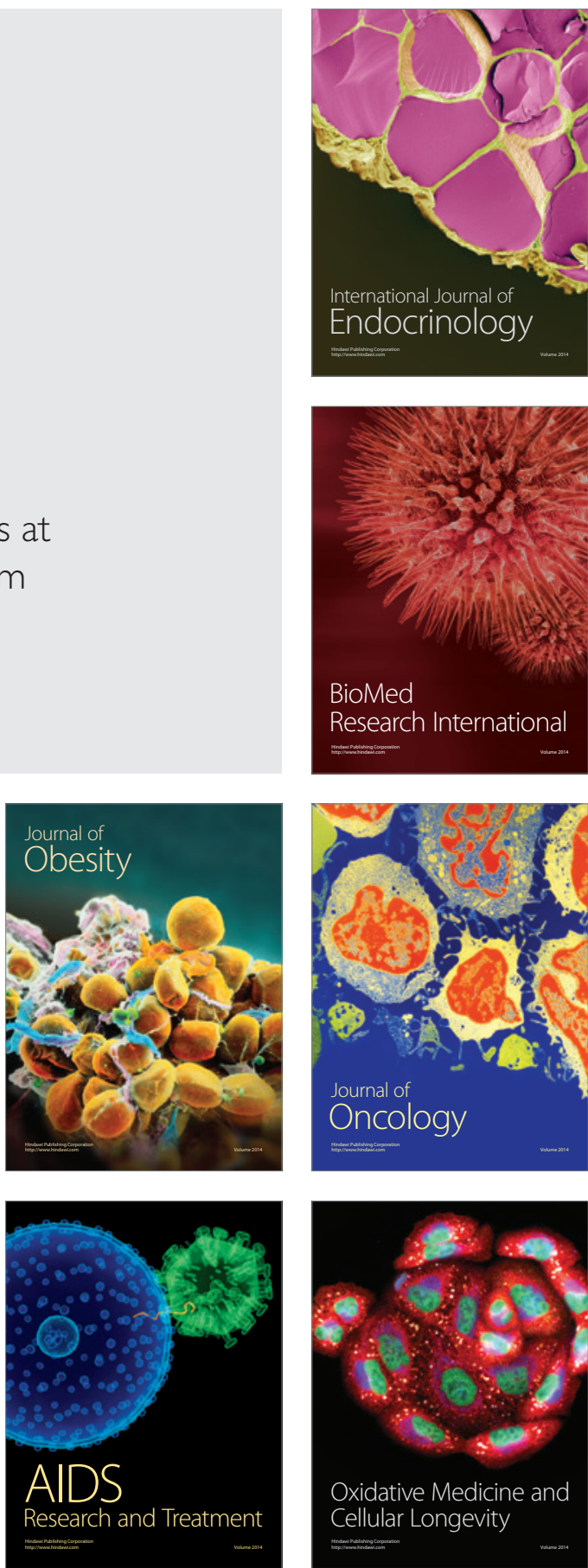